\title{
Asymptotic stability of densities for piecewise convex maps
}

\author{
by TOMOKI Inoue (Hiroshima)
}

\begin{abstract}
We study the asymptotic stability of densities for piecewise convex maps with flat bottoms or a neutral fixed point. Our main result is an improvement of Lasota and Yorke's result ([5], Theorem 4).
\end{abstract}

1. Introduction. Lasota and Yorke [5] studied the following piecewise convex maps $T:[0,1] \rightarrow[0,1]$.

(i) There exists a partition $0=a_{0}<a_{1}<\ldots<a_{r}=1$ such that the restriction of $T$ to $\left(a_{i-1}, a_{i}\right)$ is $C^{1}$ and convex; let $T_{i}$ be a continuous extension to $\left[a_{i-1}, a_{i}\right)$ of this restriction for $i=1, \ldots, r$.

(ii) $T_{i}\left(a_{i-1}\right)=0$ for $i=1, \ldots, r$.

(iii) $T_{i}^{\prime}\left(a_{i-1}\right)>0$ for $i=1, \ldots, r$.

(iv) $T_{1}^{\prime}(0)>1$.

They showed that the Frobenius-Perron operator associated with the map above is asymptotically stable in the sense of Lasota and Mackey [4], which means that the dynamics of densities is asymptotically stable and that there exists a unique invariant exact probability measure. In this paper we improve the conditions (iii) and (iv), that is, we allow $T_{i}^{\prime}\left(a_{i-1}\right)=0$ and $T_{1}^{\prime}(0)=1$ under some extra conditions.

In $\S 2$ we give some preliminary definitions. In $\S 3$ we state our main result. In $\S 5$ we prove it, using the first return map which is studied in $\S 4$.

2. Preliminaries. In this section we first give the definition of the Frobenius-Perron operator and of its asymptotic stability. Let $(X, \mathcal{F}, m)$ be a $\sigma$-finite measure space and let $T: X \rightarrow X$ be a nonsingular transformation, that is, a measurable transformation satisfying $m\left(T^{-1}(A)\right)=0$ for all $A \in \mathcal{F}$ with $m(A)=0$.

1991 Mathematics Subject Classification: Primary 28D05.

Key words and phrases: Frobenius-Perron operator, asymptotic stability, piecewise convex maps, exactness. 
Definition 2.1. The operator $P: L^{1} \rightarrow L^{1}$ defined by

$$
\int_{A} \operatorname{Pf}(x) m(d x)=\int_{T^{-1}(A)} f(x) m(d x) \quad \text { for } A \in \mathcal{F}, f \in L^{1}(m)
$$

is called the Frobenius-Perron operator associated with $(T, m)$.

By $D(m)=D(X, \mathcal{F}, m)$ we shall denote the set of all densities associated with $m$ on $X$, that is,

$$
D(m):=\left\{f \in L^{1}(m) ; f \geq 0 \text { and }\|f\|_{L^{1}(m)}=1\right\} .
$$

For $f \in D(m)$ we define a probability measure $m_{f}$ on $(X, \mathcal{F})$ by

$$
m_{f}(A)=\int_{A} f d m, \quad A \in \mathcal{F} .
$$

An $f \in D(m)$ is called a stationary density of $P$ if $P f=f m$-a.e.

Definition 2.2. $\left\{P^{n}\right\}$ is called asymptotically stable if there exists a unique density $g$ such that

$$
\lim _{n \rightarrow \infty}\left\|P^{n} f-g\right\|_{L^{1}(m)}=0 \quad \text { for all } f \in D(m) .
$$

LEMMA 2.1. If there exists a density $g \in D(m)$ such that

(2.1) $\lim _{n \rightarrow \infty}\left\|P^{n} f-g\right\|_{L^{1}(m)}=0 \quad$ for $f \in D(m)$ with $\operatorname{supp}(f) \subset \operatorname{supp}(g)$, and $m\left(X \backslash \bigcup_{n=0}^{\infty} T^{-n} \operatorname{supp}(g)\right)=0$, then $P$ is asymptotically stable.

For the proof of this, see the proof of Proposition 5.3 in [3].

Now we define exactness of a nonsingular transformation and we state a condition for exactness using Frobenius-Perron operators.

Definition 2.3. Let $(X, \mathcal{F}, \mu)$ be a probability space and $T: X \rightarrow X$ a measure preserving transformation, that is, $\mu$ is $T$-invariant. If $\bigcap_{n=0}^{\infty} T^{-n} \mathcal{F}$ is trivial, then $(T, \mu)$ is called exact.

Proposition 2.2 ([3], Proposition 2.3). If there exists $g \in D(m)$ such that (2.1) holds, then $T$ preserves the measure $m_{g}$ and $\left(T, m_{g}\right)$ is exact. Conversely, if there exists a stationary density $g$ such that $\left(T, m_{g}\right)$ is exact, then (2.1) holds.

3. Main result. Now we state the main result in this paper.

THEOREM 3.1. Assume that a map $T:[0,1] \rightarrow[0,1]$ satisfies the following conditions:

(1) There exists a partition $0=a_{0}<a_{1}<\ldots<a_{r}=1$ such that the restriction of $T$ to $\left(a_{i-1}, a_{i}\right)$ is a $C^{1}$ function; let $T_{i}$ be a continuous extension to $\left[a_{i-1}, a_{i}\right)$ of this restriction for $i=1, \ldots, r$. 
(2) $T_{i}\left(a_{i-1}\right)=0$ for $i=1, \ldots, r$.

(3) $T^{\prime}(x)>1$ for $x \in\left(0, a_{1}\right)$ and $T^{\prime}(x)>0$ for $x \in\left(a_{i-1}, a_{i}\right), i=$ $2, \ldots, r$

(4) $T_{i}^{\prime}(x)$ is an increasing function for $i=1, \ldots, r$.

(5) There exists $n_{0}$ such that

$$
\sum_{n=n_{0}}^{\infty}\left(T_{j}^{-1} T_{1}^{-n}\left(a_{1}\right)-a_{j-1}\right)<\infty
$$

for all $j$ satisfying $a_{j-1} \in \bigcup_{n=1}^{\infty} T^{n}\left(0, a_{1}\right)$.

Then the Frobenius-Perron operator associated with $T$ is asymptotically stable.

Remark 3.1. Suppose that the condition (5) of the above theorem is invalid. Then there exist no $m$-absolutely continuous $T$-invariant ergodic probability measures, but there does exist an $m$-absolutely continuous $T$-invariant ergodic $\sigma$-finite measure ([2], Theorem 1.1).

Theorem 3.1 and Remark 3.1 imply that asymptotic stability of the Frobenius-Perron operator associated with a map $T$ satisfying the conditions (1)-(4) of Theorem 3.1 is characterized by the finiteness of an $m$-absolutely continuous $T$-invariant $\sigma$-finite measure.

In the case of $T_{1}^{\prime}(0)=1$, the following corollary gives a useful criterion for the asymptotic stability of the Frobenius-Perron operator associated with $T$.

Corollary 3.2. Assume that a map $T:[0,1] \rightarrow[0,1]$ satisfies the following conditions:

(1)-(4): same as in Theorem 3.1.

(5) $T_{1}(x) \geq x+M x^{p}$ for some $M>0$ and $1<p<2$ and $T_{j}(x) \geq L(x-$ $\left.a_{j-1}\right)^{q}$ for $q<(p-1)^{-1}$ and for all $j$ with $a_{j-1} \in \bigcup_{n=1}^{\infty} T^{n}\left[0, a_{1}\right)$ and $L>0$.

Then the Frobenius-Perron operator associated with $T$ is asymptotically stable.

Remark 3.2. Under the conditions (1)-(4) of Theorem 3.1, there are no $m$-absolutely continuous $T$-invariant probability measures if $T_{1}(x) \leq$ $x+M x^{2}$ for some $M>0$ ([2], Corollary 1.1.2).

In the case of $T_{1}^{\prime}(0)>1$, the following corollary is useful.

Corollary 3.3. Assume that a map $T:[0,1] \rightarrow[0,1]$ satisfies the following conditions:

(1)-(4): same as in Theorem 3.1.

(5) $T_{1}^{\prime}(0)>1$ and $\int_{a_{j-1}}^{a_{j}} \log T(x) d m>-\infty$ for all $j$ with $a_{j-1} \in$ $\bigcup_{n=1}^{\infty} T^{n}\left[0, a_{1}\right)$ 
Then the Frobenius-Perron operator associated with $T$ is asymptotically stable.

R e mark 3.3. Suppose that the integral condition of the above corollary is invalid. Then there exist no $m$-absolutely continuous $T$-invariant ergodic probability measures ([2], Corollary 1.1.3).

This integral condition corresponds to the condition (A) for $S$-unimodal maps studied by Benedicks and Misiurewicz [1].

4. The first return map. In this section we first show how to construct an invariant measure of a given transformation from an invariant measure of the first return map and next study finiteness of the invariant measure constructed. The first return map of $T$ on $A$ is defined as $T^{n(x)}(x)$, where $n(x)$ is $\inf \left\{n \geq 1 ; T^{n(x)} \in A\right\}$. In the following lemma, let $T$ be a transformation on a measure space $(X, \mathcal{F}, m)$ and let $A \subset X$ be a measurable set with $A \subset \bigcup_{n=1}^{\infty} T^{-n}(A)$. Then the first return map is well defined.

Lemma 4.1 ([6], Lemma 2 and [2], Lemma 3.2). Let $R_{A}$ be the first return map of $T$ on $A$ and let $\mu_{A}$ be an $R_{A}$-invariant ergodic probability measure. Then the measure $\mu$ defined by

$$
\mu(D)=\sum_{n=1}^{\infty} \mu_{A}\left(A_{n} \cap T^{-n} D\right) \quad \text { for } D \in \mathcal{F}
$$

is T-invariant ergodic, where $A_{1}=A$ and $A_{n+1}=A_{n} \cap T^{-n}\left(A^{C}\right)$ for $n \geq 1$.

In the rest of this section we assume that a map $T:[0,1] \rightarrow[0,1]$ satisfies the assumptions of Theorem 3.1. Put

$$
\begin{gathered}
\alpha_{n}=T_{1}^{-n}\left(a_{1}\right) \quad \text { for } n \geq 0 \text { and } \\
\beta_{\text {in }}=T_{i}^{-1}\left(\alpha_{n}\right) \quad \text { if it exists, for } i=2, \ldots, r \text { and } n \geq 0 .
\end{gathered}
$$

Consider the first return map $R$ of $T$ on $\left[a_{1}, 1\right]$. Then $R$ can be represented in the following form. For $i=2, \ldots, r$,

$$
R(x)= \begin{cases}T(x) & \text { if } T_{i}(x)>a_{1}, \\ T^{n+1}(x) & \text { if } T_{i}(x) \in\left(\alpha_{n}, \alpha_{n-1}\right), \text { for } n \geq 1 .\end{cases}
$$

It is clear that $R(x)$ is defined except on the set of the endpoints of a countable partition of $\left[a_{1}, 1\right]$.

Lemma 4.2. Assume that there exists an m-absolutely continuous $R$ invariant probability measure whose density $g$ is bounded in the right neighborhood of $a_{j-1}$ and that there exists an integer $n_{0}$ such that

$$
\sum_{n=n_{0}}^{\infty}\left|\beta_{j, n}-a_{j-1}\right|<\infty
$$


for all $j$ satisfying $a_{j-1} \in T^{n}\left(0, a_{1}\right)$ for some $n$. Let $\mu$ be the $T$-invariant measure defined in Lemma 4.1. Then $\mu([0,1])<\infty$.

Proof. It is easy to see that there exists an integer $n_{1}$ such that

$$
A_{n}=\bigcup_{i=2}^{r}\left[a_{i-1}, \beta_{i, n-2}\right] \quad \text { for } n \geq n_{1} .
$$

There exist $n_{2} \geq n_{1}$ and $\gamma<\infty$ such that $g \leq \gamma 1_{\left(a_{j-1}, \beta_{j, n_{2}}\right)}$ for all $j$ satisfying $a_{j-1} \in T^{n}\left(0, a_{1}\right)$ for some $n$. Thus

$$
\begin{aligned}
\mu([0,1]) & =\sum_{n=1}^{\infty} \mu_{A}\left(A_{n}\right) \leq \sum_{n=1}^{n_{2}-1} \mu_{A}\left(A_{n}\right)+\sum_{i=2}^{r} \sum_{n=n_{2}}^{\infty} \mu_{A}\left(\left[a_{i-1}, \beta_{i, n-2}\right]\right) \\
& \leq \sum_{n=1}^{n_{2}-1} \mu_{A}\left(A_{n}\right)+\gamma \sum_{j} \sum_{n=n_{2}}^{\infty}\left|a_{j-1}-\beta_{j, n}\right|
\end{aligned}
$$

where $j$ satisfies $a_{j-1} \in T^{n}\left(0, a_{1}\right)$ for some $n$. Therefore $\mu([0,1])<\infty$.

Now we state upper estimates for a stationary density for piecewise monotonic maps with countable partitions which naturally arise from first return maps. Let $X$ be a union of disjoint intervals with $m(X)<\infty, S$ a map from $X$ into itself and $\left\{I_{k}\right\}$ a countable partition of $X$.

Definition 4.1. ( $\left.S, X,\left\{I_{k}\right\}\right)$ is called countable piecewise $C^{1}$ with finite images if $S$ satisfies the following three conditions:

(a) $S$ restricted to the interior of each $I_{k}$ is a $C^{1}$ function.

(b) $1 / S^{\prime}$ is of bounded variation (wherever $S^{\prime}$ is not defined we define it as the right derivative).

(c) There are only a finite number of different intervals in the collection $\left\{S\left(I_{k}\right)\right\}$.

Lemma 4.3. Assume that $S:[v, w] \rightarrow[v, w]$ is countable piecewise $C^{1}$ with finite images and $S^{\prime}(x) \geq \lambda>1$ whenever $S^{\prime}(x)$ is defined. Let $P$ be the Frobenius-Perron operator associated with $(S, m)$. Then there exists a bounded stationary density of $P$.

This lemma is an easy consequence of the proof of Theorem 1 in [6].

LEMMA 4.4. $R$ (the first return map of $T$ on $\left[a_{1}, 1\right]$ ) has an m-absolutely continuous invariant ergodic probability measure $\mu$ whose density is bounded in the right neighborhood of $a_{i}$ for $i \geq 1$ and which satisfies

$$
m\left(\left[a_{1}, 1\right] \backslash \bigcup_{n=0}^{\infty} R^{-n} \operatorname{supp}\left(\mu_{A}\right)\right)=0 .
$$


Proof. Let $\left\{I_{k}\right\}$ be the partition of $\left[a_{1}, 1\right]$ with respect to the first return map $R$. Put $\xi=\inf \left\{R^{\prime}(x) ; R^{\prime}(x)\right.$ is defined $\}$. Then it is easy to see that $\xi>0$. Put

$$
\xi_{n}=\inf \left\{\left(R^{n}\right)^{\prime}(x) ;\left(R^{n}\right)^{\prime}(x) \text { is defined }\right\} \quad \text { and } \quad \xi_{*}=\inf \left\{\xi_{n} ; n \geq 1\right\} .
$$

Clearly $\xi_{*}>0$ and we can consider the first return map $R^{*}$ of $R$ on $A^{*}$, where $A^{*}$ is the union of $I_{k}$ with $\inf _{x \in I_{k}} R^{\prime}(x)>\xi_{*}^{-1}$. It is easy to check that $R^{*}$ satisfies the assumption of Lemma 4.3. Thus $R^{*}$ has an $m$-absolutely continuous invariant probability measure $\mu_{A^{*}}$ whose density is bounded. As a consequence, $R$ has an $m$-absolutely continuous invariant probability measure $\mu_{A}$ whose density is bounded in the right neighborhood of $a_{i}$ for $i \geq 1$. (4.3) and ergodicity follow from Proposition 5.1 in [2].

5. Proof of Theorem. Let $T$ be a map satisfying the assumptions of Theorem 3.1 and let $R$ be the first return map of $T$ on $\left[a_{1}, 1\right]$. We begin the proof of Theorem 3.1 with the following lemmas.

LEMMA 5.1. There exists an $m$-absolutely continuous $T$-invariant ergodic probability measure $\mu$ such that

$$
m\left([0,1] \backslash \bigcup_{n=0}^{\infty} T^{-n} \operatorname{supp}(\mu)\right)=0 .
$$

This follows from Lemmas 4.1, 4.2 and 4.4.

Lemma 5.2. Let $P$ be the Frobenius-Perron operator associated with $T$. Then $P^{n} f$ is a decreasing function for $f$ in $D_{0}$ which is a dense subset of $D(m)$ and for sufficiently large $n$.

This is shown in the proof of Theorem 4 in [5], where the assumptions (iii) and (iv) of the introduction are not used.

LEMMA 5.3. Let $f \in D(m)$. Assume that there exists a nonnegative function $h$ such that $\|h\|_{L^{1}(m)}>0$ and that

$$
\lim _{n \rightarrow \infty}\left\|\left(P^{n} f-h\right)^{-}\right\|_{L^{1}(m)}=0 .
$$

Then there exists a stationary density $h^{*}$ such that

$$
\lim _{n \rightarrow \infty}\left\|P^{n} f-h^{*}\right\|_{L^{1}(m)}=0 .
$$

For the proof of this, see the proof of Theorem 2 in [5].

Proof of Theorem 3.1. Throughout the proof $\|\cdot\|$ stands for $\|\cdot\|_{L^{1}(m)}$. Let $g$ be the density corresponding to the $m$-absolutely continuous $T$-invariant ergodic probability measure of Lemma 5.1 and let $c$ be an arbitrary positive constant. First, we prove that

$$
\lim _{n \rightarrow \infty}\left\|P^{n} f-g\right\|=0 \quad \text { for } f \in D(m) \text { with } f \leq c g .
$$


Let $z$ be a positive constant satisfying

$$
\int_{0}^{z} c g d m<1 / 2
$$

Then

$$
P^{n} f \geq \frac{1}{2} \cdot 1_{(0, z)} \quad \text { for } f \in D_{0} \text { with } f \leq c g .
$$

In fact, if not, it follows from Lemma 5.2 that there exists $y \in[0, z)$ such that

$$
1=\int_{0}^{y} P^{n} f d m+\int_{y}^{1} P^{n} f d m \leq \int_{0}^{z} c g d m+\frac{1}{2}(1-y)<1,
$$

which is impossible and (5.2) is proved. (5.2) and Lemma 5.3 imply that

$$
\lim _{n \rightarrow \infty}\left\|P^{n} f-h^{*}\right\|=0 \quad \text { for } f \in D_{0} \text { with } f \leq c g .
$$

Since $\left(T, m_{g}\right)$ is ergodic, we have $h^{*}=g$. Thus we obtain (5.1).

Next, we prove that

$$
\lim _{n \rightarrow \infty}\left\|P^{n} f-g\right\|=0 \quad \text { for } f \in D(m) \text { with } \operatorname{supp}(f) \subset \operatorname{supp}(g) .
$$

Put $f_{c}=\min (f, c g)$. Then $f=\left\|f_{c}\right\|^{-1} f_{c}+r_{c}$, where $r_{c}=\left(1-\left\|f_{c}\right\|^{-1}\right) f_{c}+$ $f-f_{c}$. Since $\operatorname{supp}(f) \subset \operatorname{supp}(g)$, we have $\lim _{c \rightarrow \infty} f_{c}(x)=f(x)$ for each $x$. Hence $\left\|f_{c}-f\right\| \rightarrow 0$ and $\left\|f_{c}\right\| \rightarrow\|f\|=1(c \rightarrow \infty)$. Thus, for any $\varepsilon>0$ we can find a constant $c$ such that $\left\|r_{c}\right\|<2^{-1} \varepsilon$. Since $\left\|f_{c}\right\|^{-1} f_{c}$ is a density bounded by $c\left\|f_{c}\right\|^{-1} g$, it follows from the first part of the proof that $\left\|P^{n}\left(\left\|f_{c}\right\|^{-1} f_{c}\right)-g\right\| \leq 2^{-1} \varepsilon$ for sufficiently large $n$. Therefore, $\left\|P^{n} f-g\right\|<\varepsilon$ for sufficiently large $n$.

By (5.3) and Lemma 5.1, Lemma 2.1 finishes the proof.

The following lemma is used to prove Corollary 3.2 and is easily verified.

LEMMA 5.4. Let $T:[0, a] \rightarrow[0,1]$ be a continuous strictly increasing function with $T(0)=0$, where $a$ is a positive constant. If $T(x) \geq x+M x^{p}$ for some $p>1$ and $M>0$ on $(0, a]$, then there exists a $k$ such that

$$
T^{-n}(a) \leq\left(k+2^{-1}(p-1) M n\right)^{1 /(p-1)} \quad \text { for all } n .
$$

Proof. Put $\tau(x)=x+M x^{p}$. Since $x<\tau(x) \leq T(x)$, we have $T^{-n}(a) \leq \tau^{-n}(a)$. By an elementary calculation, we have

$$
\tau\left(\frac{1}{n^{1 /(p-1)}}\right) \geq \frac{1}{\left(n-2^{-1}(p-1) M\right)^{1 /(p-1)}} \quad \text { for large } n .
$$

Therefore, for a $k$ with $k^{1 /(1-p)} \geq a$, we get

$$
T^{-n}(a) \leq \tau^{-n}(a) \leq \tau^{-n}\left(\frac{1}{k^{1 /(p-1)}}\right) \leq \frac{1}{\left(k+2^{-1}(p-1) n M\right)^{1 /(p-1)}} .
$$


Proof of Corollary 3.2. By Lemma 5.4 the conditions (1)-(3) and (5) of Corollary 3.2 imply the condition (5) of Theorem 3.1.

The following lemma is used to prove Corollary 3.3.

LEMma 5.5. Let $T:[a, b] \rightarrow[0, T(b)]$ be a strictly increasing $C^{1}$ function with $T(a)=0$. If

$$
\int_{a}^{b} \log T(x) d m>-\infty
$$

then for $0<\alpha<1$

$$
\sum_{n=1}^{\infty} T^{-1}\left(\alpha^{n}\right)<\infty .
$$

For the proof of this lemma, see Lemmas 1 and 2 in [1].

Proof of Corollary 3.3. By Lemma 5.5 the conditions (1)-(3) and (5) of Corollary 3.3 imply the condition (5) of Theorem 3.1.

\section{References}

[1] M. Benedicks and M. Misiurewicz, Absolutely continuous invariant measures for maps with flat tops, Publ. Math. IHES 69 (1989), 203-213.

[2] T. Inoue, Weakly attracting repellors for piecewise convex maps, preprint.

[3] T. Inoue and H. Ishitani, Asymptotic periodicity of densities and ergodic properties for nonsingular systems, Hiroshima Math. J. 21 (1991), 597-620.

[4] A. Lasota and M. C. Mackey, Probabilistic Properties of Deterministic Systems, Cambridge University Press, 1984.

[5] A. Lasota and J. A. Yorke, Exact dynamical systems and the Frobenius-Perron operator, Trans. Amer. Math. Soc. 273 (1982), 375-384.

[6] G. Pianigiani, First return map and invariant measures, Israel J. Math. 35 (1980), $32-48$.

[7] V. A. Rokhlin, Exact endomorphisms of a Lebesgue space, Amer. Math. Soc. Transl. Ser. (2) 39 (1964), 1-36.

DEPARTMENT OF MATHEMATICS

FACULTY OF SCIENCE

HIROSHIMA UNIVERSITY

HIGASHI-HIROSHIMA

HIROSHIMA 724, JAPAN

E-mail: INOUE@HUMPTY.MIS.HIROSHIMA-U.AC.JP 